\title{
The composition and characteristics of conscious environment
}

\author{
Ruiyu Zhu*1 \\ *Jiangnan University, Wuxi, P.R. China.
}

\begin{abstract}
Although we are faced with a complicated and objective world, the conscious environment is the world that the spiritual self can directly perceive; the real self lives in the conscious environment. This artical discusses the composition and characteristics of human conscious environment. First, this article proposes that the conscious environment is composed of five aspects: conscious scene, experience circuit, emotional label, concept, and values. Second, it provides a discussion of the thinking, reasoning, beliefs, and the theory of use and disuse upon which the further growth of cognition depends. Finally, it explains other characteristics of cognition. For example, knowing determines perception, the easily imprisoned soul, its assimilation, and the limitations of one's own cognition. Moreover, the importance of emptying is recognized. This part of the article presents the concept of conscious environment for the first time and systematically discusses the influencing factors and characteristics of the human conscious world. Its purpose is to provide a preliminary and systematic understanding of the human spiritual world and to provide a theoretical basis for the following concepts: the natural driving force, the underlying thinking about life, and the rational reconstruction of the spiritual world.
\end{abstract}

Perhaps we are constantly undergoing changes in different objective environments every day. However, our inner spiritual world is relatively stable and unchanged. In fact, the spiritual self is living in the conscious environment; the conscious environment is the world that we directly perceive. For most people, the conscious environment is passively shaped by the environment, rather than being actively constructed by the individual. The conscious environment in our brain is independent of the external environment. This protects us from being disturbed and controlled by external fluctuations, language, or reason. This permits the spiritual self to enjoy a relatively stable living environment. Our emotions and needs affect the state of consciousness. The basic materials of conscious environments mainly include conscious scenes, experience circuits, emotional tags, concepts and values. The existing consciousness forms our subconsciousness and constrains the space and content that are of concern. The main components of the conscious environment and the rules that regulate it are discussed below.

\section{The basic composition of the conscious environment}

\section{1) Conscious scene}

The consciousness in our mind is derived from the memory of our life experiences. Humans mainly have five kinds of receptors: sight, sound, smell, taste, and touch. They constantly are

\footnotetext{
${ }^{1}$ This is a working document which has not been peer reviewed yet. Please address any comment or remark to ry_zhu@hotmail.com or ry_zhu@sina.com.
} 
used to perceive the external world. The signals obtained by these five receptors have become the most basic material of the conscious scene in our brain - the world of our consciousness. Visual signals prove to be a particularly important source of signals, followed by auditory signals. I call the information world in the brain that is made available to the primary sensory organs the "conscious scene." The conscious scene is the material basis of the spiritual world. In fact, not every individual fully possess these receptors. For example, congenital blindness, or the absence of visual nerves, prevents perception of colors in the real world. The absence of the optic nerve turns tactile sense into the primary organ of perception of the shape of the objective world. People who experience the onset of blindness have previous memories of sight. Therefore, they are able to perceive the colors of a blue sky, white clouds, stars, or the moon; people blind from birth can only perceive through abstract thinking.

Experience determines the consciousness scene. If we have not experienced it, we cannot know what the real consciousness scene is. For example, a congenital blind person has never seen color. Therefore, they can never have color in his conscious scene. People who acquired blindness have seen color and are able to have color in their conscious scene. The conscious scene is just a perception of the external objective world and reflects the message of the external objective world. Our brain evaluates the information obtained by various receptors as a whole, thus forming an experience circuit based on that overall knowledge.

\section{2) Experience circuit}

The conscious scene is only a partial perception of the receptor, a perception from the perspective of the observer. The consciousness that integrates the subject of feeling is called experience; experience is the comprehensive perception of subject participation. For the observer, an object and me are discrete entities that are not related. However, the observed object is integrated with the experiencer. The formation and acquisition of the experience circuit is the basis for humans to experience empathy for people and to form an understanding of the truths related to people. For humans or animals, there is an experience circuit of external signals. People connect the experience circuit with language, thereby giving the language a sense of experience in the body. If there is no such experience in the mind, these words or natural signals cannot construct the corresponding perception. For example, men cannot experience women's physiological cycles. Nor can women experience the feeling of men shaving their beards. In addition to their own feelings, there are various experiences of external things. These experiences provide a basic imaginative space for individuals to further perceive external signals. Therefore, people need to experience a variety of scenes. They also need to experience as many psychological setbacks as possible. Such experiences can become the basis for increased processing of signals in the brain.

\section{3) Emotional tags}

Following the internalization of all types of information from the receptor into one's own experience circuit, a person usually conducts emotional assessments to connect their experience with various emotions and mark external stimuli. The establishment of the external signal experience circuit and the attachment of emotional tags are performed simultaneously. However, essentially they are discrete steps. Emotional labels are strong signals that we can feel. It is very easy to transmit signals through language. Yet that step is part of a process that we are able to actively adjust. Obviously, factors such as living environment and experience will cause us to formulate different emotions for the same thing. Such emotions are natural and spontaneous. They are a manifestation of the mental state of things we observe. Nevertheless, there are many shortcomings. The emergence of emotions only plays a role in recognition, and they can 
be wrong. Therefore, it is easy to passively establish many negative connections and kidnap the conscious environment, yet remain unaware of that having occurred. The proactive correction of negative emotional labels is an effective way to avoid psychological difficulties.

The conscious scene, the experience circuit and the emotional tags together constitute the emotional part of spiritual world activity. Perceptual materials are the basis and premise of rational activity. The foundations of rational thinking are concepts and values. The formation of concepts is the basis for an ability to make distinctions and create an abstract description of objective existence, which is the most basic material on which rational thinking depends. Values represent a subjective evaluation of objective existence. They are the basis for, and premise of, logical deduction. The influence of receptors on thinking activities is a bottom-up path, whereas the adjustment of thinking activities on receptors is a top-down path. Bottom-up represents a positive and top-down a negative feedback adjustment mode.

\section{4) Concepts}

A concept is an abstract description of objective existence and forms the basis of cognition and rational thinking. "Mangosteen is delicious". If one has not seen mangosteen, one cannot form a concept. It would be difficult to achieve the inner experience of mangosteen as delicious. Therefore one could not truly understand this sentence. A broader experience with real life would be needed. Multiple experiences are used to acquire rich conceptual elements in thoughts, thereby providing a basis for further cognitive construction. The concept of things that can be intuitively perceived can be obtained through practical activities; it is extremely difficult to comprehend abstract concepts that have not been perceived or are entirely new. A typical example is the punctuation mark. Perhaps when one sees the punctuation mark in the article, we think it is a very simple and easy invention. However, looking back thousands of years, it is apparent that China never invented a symbol that can separate sentences. The inner constraint of consciousness is so powerful that every step of breaking through the boundaries of human consciousness is very difficult. Logical thinking is a continuous process, whereas a concept implies a spontaneous invention made from scratch. The abstract concept itself cannot be grasped through rational thinking. Concept breakthroughs are the foundation of innovation and crucial for the generation of new knowledge. Conceptual innovation is more important than rational thinking.

\section{5) Values}

Values are subjective evaluations of the objective world and the basis of rational thinking. There are two main sources of values. The first is the environment and the second is experience. "Faith will exist, if there is no faith, there will be no existence" is the most important rule on the spiritual level. Is the world that we see truly real? With the continuous improvement of cognition, an understanding of the outside world is constantly changing. Each person's characteristics, as well as their physiological conditions, are different. Even for the same person, there can be very big differences. The existence of these factors necessarily creates differences in consciousness. Belief is the basis for a further evolution of thinking. Moreover, belief is equivalent to the determination and existence of signals and provides support for further development.

A certainty in values will further develop into thinking and behaviors. Prior to a living environmental testing, a person unconsciously thinks and acts according to inertia. People transform the environment of consciousness to ensure that values can persist, impeding the entry of different values. Therefore, it makes sense to be stubborn and conservative as one ages. The influence of values on human cognition is reflected in many aspects. For example, why are 
children simple? Children are simple because they experience relatively few situations. It is easy to use simple criteria to evaluate anything. In fact, this is true for any person who is not mature. They are able to measure everything with simple-especially formulaic-standards. However, when people experience a variety of realities that do not meet the standard established, they come to understand that the standard cannot fully account for reality. This creates a situation in which people minimize their acceptance of the standard and embrace alternatives to it. The real world is extremely complex, and complexity will evolve into rich and diverse life content. After a value is formed, it further develops a system of values and enhances an understanding of the world. This is a self-reinforcing positive feedback process. This growth is not only reflected in an understanding of external things, but also in judgment, certainty, and confidence.

A person's true self lives in their conscious environment and is impacted by existing concepts and cognitive structures. Values need to be verified by the environment. If there is a value rooted in culture, it cannot be tested. It can be seen from the relationship between the simultaneous movement of values and the environment of consciousness. We may achieve internal and external consistency through changes in the external environment or changes in the internal values, thereby producing psychological pleasure. Additionally, values impact attention, and the establishment of the conscious environment. Therefore, the importance placed on values is strengthened and amplified.

\section{Cognitive growth}

\section{1) Thinking and establishing contacts}

The purpose of thinking is to form deeper connections and master external situations. The act of thinking forms internal experience chains, which increase understanding. Understanding is an in-depth thinking process. The more knowledge and understanding reserves one has, the greater one's understanding.

Understanding forms underlying logical connections. The deeper the understanding, the greater the sympathy chain that can be formed. For example, take information such as apples, oranges, dogs, cats, bugs, viruses, and DNA. If I can understand fruits, I can connect apples and oranges. If I am able to understand eukaryotic cells, I can connect apples, oranges, dogs, cats, and bugs through them. If I am able to understand nucleic acids, I am able to link all of the above information. The formation of this deep network creates a foundation for an orderly architecture of concepts and a deep internal connection with objective matter.

2) The truth exists in the connection. Understanding the truth demands an activation of the experience circuit.

Truth describes the interaction between multiple elements. Truth itself cannot be expressed through specific elements. However, in real life, our understanding of truth often comes from memory. This kind of memory is only written and existing as a complete message. If a person has not had relevant experience, they are only able to make judgments of right and wrong. They are not able to stimulate the inner experience nor obtain or understand truth. When teaching truth, we must be able to stimulate the listeners experiences so that they may truly understand the truth. Truth can only exist in connections or interactions among specific things. When the truth itself is regarded as a concrete static existence, it becomes rigid and cannot be understood or truly grasped. As the Tao Te Ching says, "The Tao that can be told of is not the eternal Tao; the name that can be named is not the eternal name." 
For people in underdeveloped areas, the most important reason for underdevelopment is that they lack experience and are unable to obtain a deeper understanding of the world. Even if they receive indoctrination, it is difficult for them to form an inner experience that could produce real change. Therefore, the best education is experience. In the future, games and virtual reality may become a convenient way to increase people's inner experience and improve people's cognition.

3) Trust, the definitive basis for the growth of the spiritual world.

"Faith will exist. If there is no Faith, there will be no existence" is the most important rule to follow on the spiritual plane. Of course, this belief may be unrealistic. It may be rejected in the course of further practice, resulting in disbelief and the possible abandonment of the spiritual world. Faith is the certainty of establishing connections. It provides a foundation and materials for the spiritual world. It also is the basis for further structuring of the spiritual world. A specific belief can serve to the iteration and development of the spiritual world.

\section{4) Use and disuse}

The idea of "use and disuse" was first proposed by the French biologist Lamarck, who systematically expounded the doctrine of chemistry in "Philosophy of Animals" (referred to as "Lamarck's doctrine" by later generations). The direct influence of the new environment, together with changes in habits, the development of certain frequently used organs, and the gradual degradation of infrequently used organs result in "use and disuse." The material basis for thinking is the brain. As a living body, the most basic principles and underlying logic are adaptability and the principle of "use and disuse". It is easier to strengthen emotions with emotions and reasons with reasons. Therefore, it also is necessary to distinguish between emotional feelings and reason. People's egos (the "I") are more sensitive to sensibility, as opposed to rationality. Therefore, uncertainty and defensiveness with regard to sensibility will be stronger. Rationality excludes the emotional self, making it more stable. The key of use and disuse is to let everyone understand the importance of training and growth. The idea that "faith will exist" emphasizes the importance of recognition of good ideas and the importance of growth.

\section{Other features}

1) What we can see depends on what we know

The world we can see is just the world we can understand, not the real world. How are we able to perceive this world? The world we perceive is undoubtedly the result of the interaction between the external objective world and the brain. Many people may think that the idea that "what you know determines what you see" is an idealistic point of view. However, this is one of the objective laws of the spiritual arena. Our understanding of external phenomena requires a process of development and evolution. For a hidden or undetectable entity, there must exist a corresponding concept in the mind for us to be able to observe it. Our observation of the outside world is formed in a way that resonates. Resonance determines that there must be a corresponding basis for things external to our minds. The essence of external observations is electromagnetic waves. Therefore, resonance proves central to our capability of perception. With no inherent signal, it is difficult for resonance to be created. Consequently, it would be difficult to observe it. We need to constantly raise our cognition, and only in this way can we perceive a brighter world. 
How to achieve resonance? After the brain has formed a concept, a person receives the corresponding signal. If the concept is systematic, there will be differences in primary and secondary positions within the system. Only when there is a resonance can we see the outside world. Without learning, the lack of corresponding concepts in the brain makes it difficult to accept and understand. The process of understanding is also the process of further digesting external signals within one's own logical structure and experience. The improvement of cognitive ability is gradual and slow; it is the process of growth and change. A person may fairly easily accept and process novel phenomena. However, it proves harder to learn about something for which an interpretation already has been formulated. That requires a reworking of the connections that already exist in the brain. The affirmation or determination of external things requires constant strength and an open heart. Only in this way may cognitive judgment create a more stable material foundation in the brain so as to we produce more precise judgments about external phenomena. The result is greater confidence. Such self-confidence is healthy and positive, not blind. Many questions about life cannot be reduced to an understanding of how things are external and whether or not they have changed, but whether the internal cognitive structure truly reflects that which is external. Self-reinforcement training serves to improve an ability to better resonate with the outside world.

\section{2) Limitations of human cognition}

Everyone's understanding has great limitations. Both the time and space devoted to perception, as well as the angle of observation, are limited. Due to differences in time and space, physiological basis, and life cycle nodes, there exists an asymmetry of information and perception. Only through communication can one resolve such differences, achieve emotional stability, use rational speculation to grasp the rules underlying phenomena, and achieve symbiosis through love and tolerance. Through these means, self-imposed restrictions may be shed and cognitive life blossom.

\section{3) The spiritual world imprisoned by the body}

Initially, all human nerve cell tissues exist for human survival. The development of the cerebral cortex is the final stage. Perceptual thinking is primarily controlled by the limbic system of the brain. The control of the brain by the limbic system is direct and powerful. The limbic system directly regulates the control of blood flow from the heart. The function of the brain is based on the stability of the heart. If the blood flow is unstable, the brain will not be able to engage in rational thought. Perceptual thinking belongs to the most basic control range of the body, it is difficult to rid oneself of the restraints imposed by emotions. Most of the time a person can think effectively because physiological impulses of various receptors control the brain and not the other way around. Therefore, the mind is easily imprisoned by the body.

Moreover, physiological needs arise naturally. They are easily stimulated, and often must be met. Often humans are unconsciously and passively controlled by desire. The intensity of desire's control over thought may even exceed its control over life itself. For example, every year many people commit suicide or risk their lives.

\section{4) The assimilation effect}

The conscious environment of the observed world will become a part of our soul. Subconsciously, people wish to change others to become the same as them or to meet their expectations. They evaluate the outside world based on the conscious environment. The recognized part of the conscious environment will be affirmed and the unrecognized part will be denied. This occurs subconsciously. Through a constant process of recognition and evaluation, 
people tend to mold others in their surroundings, especially children. People who lack self-reflection are more likely to assimilate. Often they are unable to recognize the problems with the environment. The assimilation effect virtually intensifies problems in the environment. This also is the reason why weak countries experience stronger constraints intensified by the inherent weakness that already exists. The role of assimilation strengthens retrograde ideas and makes it difficult for individual or social changes to occur.

5) Emptiness for maximum spiritual energy

Spirit reflects the connections among entities. When there are more things that exist in the spiritual world, there will be greater constraints. The spiritual world thrives on less content. Therefore, emptying actually removes spiritual restraints; the brain has access to more energy. Emptiness also implies infinite possibilities. The act of emptying one's mind also represents one of the primary practices of many traditional health care programs. Additionally, the spiritual world is characterized by the tenet of receiving what is imagined. We know that emptiness is ubiquitous and all-encompassing; it is the main part of the universe and possesses great energy. When one pays attention to emptiness, the reward is great energy. Meditation takes on a special meaning when practiced with an empty mind. 